\title{
The Wind Momentum-Luminosity Relationship: blue supergiants in the spiral galaxy NGC 300
}

\author{
Fabio Bresolin, Rolf-Peter Kudritzki \\ Institute for Astronomy, University of Hawaii at Manoa, \\ 2680 Woodlawn Drive, Honolulu, HI 96822, USA \\ Wolfgang Gieren \\ Universidad de Concepción, Departamento de Física, \\ Casilla 160-C, Concepción, Chile
}

Abstract. We have carried out the wind analysis of six A-type supergiants in NGC 300. The derived Wind Momentum-Luminosity Relationship is compared with that of Galactic and M 31 blue supergiants and with theoretical models.

\section{Introduction}

The theory of radiatively driven winds predicts a relationship between the modified wind momentum and a power of stellar luminosity:

$$
\log \left(\dot{M} v_{\infty} R\right)=x \log L+\text { const }
$$

where $\dot{M}$ is the mass-loss rate, $v_{\infty}$ the wind terminal velocity and $R$ the stellar radius. Empirical verifications of this Wind Momentum-Luminosity Relationship (WLR) have so far been carried out for O-type stars in the Milky Way and the Magellanic Clouds by Puls et al. (1996), and for Galactic and M 31 B- and A-type supergiants by Kudritzki et al. (1999). The brightest A-type supergiants in galaxies are extremely bright, with $M_{V}$ up to $-10 \mathrm{mag}$, making the WLR a potential extragalactic distance indicator. However, extensive work still needs to be carried out on the empirical calibration of the relation and on the theoretical modeling of the effects of stellar metallicity and spectral type.

\section{A-type supergiants in NGC 300}

We have carried out a spectroscopic survey of blue supergiants in the Sculptor galaxy NGC 300 (Cepheid distance $\sim 2 \mathrm{Mpc}$ ) with multiobject spectroscopy at $5 \AA$ resolution with VLT-Fors (Bresolin et al. 2002). These data enable us to determine spectral type, metallicity, effective temperature and reddening of the confirmed blue supergiants. In addition, the stellar mass-loss rate can be derived from the $\mathrm{H} \alpha$ line profile. 


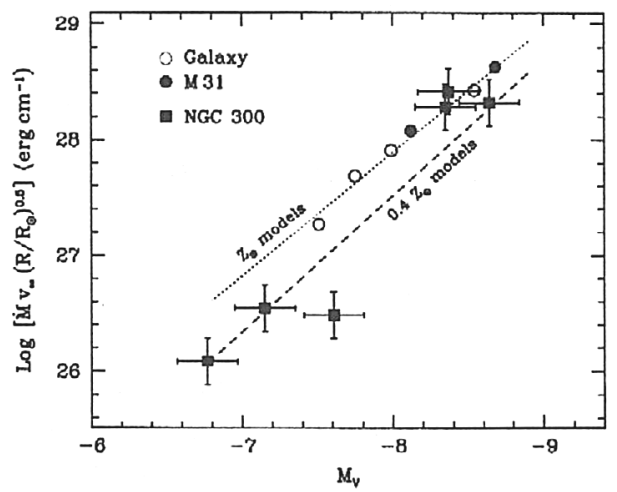

Figure 1. The WLR of the six A-type supergiants analyzed in NGC 300, compared with stars in the Galaxy and M 31. Theoretical models at solar and 0.4 solar metallicity are also shown.

For our analysis we have selected six late B- to early A-type (B8 - A2) supergiants, whose spectra appear uncontaminated by nebular emission. Stellar gravities and mass-loss rates have been measured by means of profile fits to the $\mathrm{H} \gamma$ and $\mathrm{H} \alpha$ lines, adopting the non-LTE line formation code of Santolaya-Rey, Puls \& Herrero (1997). The modified wind momentum has been derived from the measured $\dot{M}$ and the known distance (which, together with the apparent magnitude, provides the stellar radius), and by assuming a terminal velocity $v_{\infty}=150 \mathrm{~km} \mathrm{~s}^{-1}$. This is currently the major source of uncertainty, since $v_{\infty}$ cannot be measured at the resolution of our spectra.

We show in Figure 1 the resulting WLR for the six supergiants in NGC 300 (squares), compared with the relationship for an equal number of A-type supergiants in the Milky Way and M 31 (circles; from Kudritzki et al. 1999). Also plotted are new theoretical models accounting for the metallicity dependence of the wind momentum, calculated for solar and 0.4 solar metallicity. The latter value corresponds to the oxygen abundance derived from the $\mathrm{H}$ II region galactic radial abundance gradient (Zaritsky, Kennicutt \& Huchra 1994) and measured at the mean galactocentric distance of our supergiant sample $(\sim 5 \mathrm{kpc})$. We find a reasonable agreement between the data points and the models, which predict $\dot{M} v_{\infty} R \propto Z^{\alpha}$, with $\alpha \simeq 0.8$. Our next step will be to further investigate the systematics of the WLR in terms of stellar parameters $\left(\log g, T_{\text {eff }}\right.$, metallicity).

\section{References}

Bresolin, F., Gieren, W., Kudritzki, R.-P., Pietrzyński, G., Przybilla, N., 2002, ApJ 567, 277

Kudritzki, R.-P., Puls, J., Lennon, D.J., Venn, K.A., Reetz, J., Najarro, F., McCarthy, J.K., Herrero, A. 1999, A\&A 350, 970

Puls, J., Kudritzki, R.-P., Herrero, A., et al. 1996, A\&A 305, 171

Santolaya-Rey, A.E., Puls, J., Herrero, A. 1997, A\&A 323, 488

Zaritsky, D., Kennicutt, R.C., Huchra, J.P. 1994, ApJ 420, 87 